\title{
Jóvenes en México: ¡existen diferencias entre hombres y mujeres en su inicio sexual y uso del condón?
}

\section{Young in Mexico: are there differences between men and women in your sexual initiation and condom use?}

\author{
Catherine Menkes-Bancet*, David de Jesús-Reyes**, \\ Itzel A. Sosa-Sánchez* \\ *Centro Regional de Investigaciones Multidisciplinarias de la Universidad Nacional \\ Autónoma de México, México \\ **Instituto de Investigaciones Sociales de la Universidad \\ Autónoma de Nuevo León, México
}

\section{Resumen}

El artículo analiza las características del inicio sexual de los jóvenes mexicanos de 12 a 29 años de edad, así como los factores que se relacionan con mayor uso de métodos anticonceptivos, en particular del uso del condón, enfatizando el rol de las desigualdades de género sobre las prácticas sexuales. Se analizó el inicio sexual de los jóvenes en distintos contextos, comparando entre sexos. También se estudió el uso de los métodos anticonceptivos en la primera relación sexual. Para conocer los factores sociodemográficos relacionados con el uso de un preservativo, se estimaron dos modelos de regresión logística estratificados por sexo. Los datos provienen de la Encuesta Nacional de la Juventud 2010.* Los resultados muestran diferencias por sexo tanto en la edad de inicio sexual, como en las características de la pareja y en los significados dados al inicio sexual. Asimismo, los jóvenes con estereotipos de género más tradicionales incrementan la probabilidad de inicio sexualmente a edades más tempranas, y tienen menor probabilidad de protegerse de un embarazo no planeado y de una infección de transmisión sexual.

Palabras clave: Sexualidad, género, jóvenes en México, uso de condón.

Abstract

The article analyzes the characteristics of the sexual onset of Mexican youth between 12 and 29 years of age, as well as the factors that are related to the greater use of contraceptive methods, in particular the use of condoms, emphasizing the role of gender inequalities over sexual practices The sexual onset of young people in different contexts was analyzed, comparing between sexes. The use of contraceptive methods in the first sexual intercourse was also studied. To know the sociodemographic factors related to the use of a condom, two logistic regression models stratified by sex were estimated. The data comes from Encuesta Nacional de la Juventud 2010.* The results show differences by sex both in the age of sexual onset, as in the characteristics of the couple and in the meanings given at the sexual onset. Likewise, young people with more traditional gender stereotypes increase the likelihood of starting sexually at earlier ages, and are less likely to protect themselves from an unplanned pregnancy and a sexually transmitted infection.

Keywords: Sexuality, gender, youth in Mexico, condom use.

\footnotetext{
* Encuesta Nacional de la Juventud 2010 realizada por el Instituto Mexicano de la Juventud y la
} Universidad Nacional Autónoma de México (IMJUVE, 2011). 


\section{INTRODUCCIÓN}

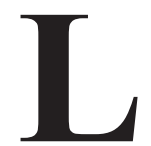

a salud sexual y reproductiva de las y los jóvenes mexicanos en general y de las y los adolescentes en particular, constituye un tema central de la agenda académica y política en México (Juárez y Gayet, 2005; Rodríguez, 2014). En países en vías de desarrollo como México, el tema cobra mayor relevancia si se toma en cuenta que en la última década como parte de una política de población de corte conservador, las adolescentes se han visto comparativamente rezagadas en el descenso de sus niveles de embarazo y de fecundidad en relación con otros grupos de edad. Así, la incidencia del embarazo adolescente en México es un problema importante no sólo de salud sexual y reproductiva sino de derechos sexuales, reproductivos y humanos.

$\mathrm{Al}$ respecto, diversos estudios han señalado que el embarazo en edades tempranas puede limitar las opciones de desarrollo personal de los adolescentes en determinados contextos socioculturales (Romero, 2006). Para el caso mexicano las estimaciones sugieren que las gestaciones de las adolescentes de 15 a 19 años, constituyeron 17.5 por ciento de la tasa de embarazo del total de mujeres de 15 a 49 años en 2008, mientras que en 1991 este porcentaje fue de 12.1 por ciento, además se ha observado un ligero aumento en los últimos años, ya que la tasa específica de 15 a 19 años pasó de 68 a 85 embarazos por cada mil mujeres, entre 2005 y 2013 (Menkes y Serrano, 2010; Menkes, 2016).

Adicionalmente, diversos estudios han señalado que el embarazo en edades tempranas puede representar un riesgo biopsicosocial para la madre y el recién nacido y una mayor mortalidad materno-infantil (Fraser et al., 1995). Así, el riesgo de muerte por complicaciones en el embarazo es mucho mayor en las adolescentes ya que las mujeres menores de 16 años tienen cuatro veces más probabilidades de morir a causa del embarazo que las mujeres de entre 20 y 30 años. Igualmente, cuando se trata de un embarazo no deseado, los trabajos muestran que existe un riesgo social mayor para el binomio madre-hijo asociándosele con la elección del aborto, el escaso cuidado de la salud durante el embarazo, complicaciones perinatales, el abuso infantil y algunos problemas en el desarrollo de las y los niños (Ulanowitcz et al., 2006).

Paralelamente la sexualidad adolescente también ha adquirido mayor visibilidad en la agenda académica y política, debido al reciente incremen- 
Jóvenes en México: ¿existen diferencias entre hombres y mujeres en su inicio sexual y uso del condón? / C. MENKES et al.

to de las infecciones de transmisión sexual (ITS) en esta población. Desde los años noventa, las estadísticas sobre SIDA han sugerido que un porcentaje significativo de los nuevos casos ocurre en personas que habían sido infectadas durante la adolescencia (Cáceres, 1999). Lo antes mencionado, contribuyó a resaltar la importancia de generar evidencias empíricas que permitan saber más sobre la sexualidad, de la dinámica y la lógica de las prácticas sexuales y reproductivas en grupos poblacionales de adolescentes y jóvenes, enfatizando en las relaciones de género y poder, así como en los factores socioeconómicos y culturales que determinan dichos comportamientos, pues es muy evidente que dichos comportamientos no se pueden generalizar (Sosa y Menkes; 2003; Jones, 2010; De Jesús y González, 2014).

Los estudios demográficos y sociológicos han enfatizado el uso reducido de anticonceptivos entre los adolescentes, al tiempo que la demanda insatisfecha de los mismos en esta población continúa siendo elevada (Juárez et al., 2010; Mendoza et al., 2009). Al respecto según datos recientes, mientras que 75.6 por ciento de las mujeres de 15 a 49 años sexualmente activas utilizaba algún método anticonceptivo en 2014, únicamente 59 por ciento de las adolescentes de 15 a 19 años se protegió con algún un anticonceptivo (Menkes, 2016).

$\mathrm{Al}$ respecto se ha señalado reiteradamente, la existencia de una brecha entre la información que tienen las personas sobre las medidas para prevenir y espaciar el embarazo y protegerse del contagio de infecciones de transmisión sexual, frente a las actitudes y prácticas de esas personas, lo cual en los adolescentes esta brecha es particularmente importante (Szasz, 1998). Por ejemplo, en cuanto a la protección sexual, si bien se han dado grandes avances en el uso de métodos anticonceptivos en la primera relación sexual, ya que según la información que proporcionan las encuestas la proporción de mujeres adolescentes de 12 a 19 años que no se protegieron de 2000 a 2012 se redujo de 79.1 a 33.1 por ciento (Instituto Nacional de Salud Pública, 2000 y Instituto Nacional de Salud Pública, 2012), todavía una proporción importante de jovencitas estás expuestas a un embarazo no planeado y a las infecciones de transmisión sexual en su primera relación sexual.

Cabe mencionar que diversos estudios en el tema, han dado origen a una reflexión en torno a los vínculos entre las relaciones y desigualdades de género y los comportamientos y actitudes frente a la salud y a la sexualidad. Desde esta perspectiva, se han propuesto algunas dimensiones analíticas para profundizar en el estudio de la forma en que la construcción 
de las identidades y relaciones de género y las desigualdades de acceso al poder se constituyen como un factor de riesgo en torno a la salud y a la sexualidad tanto para las mujeres como para los hombres a partir de distintos mecanismos: la construcción simbólica del cuerpo, la asignación genérica del concepto de cuidado, la construcción social del riesgo, la relación entre la afirmación de la identidad de género, el comportamiento sexual y las decisiones sobre reproducción (De Keijzer, 1999; Sosa, 2005; Jones, 2010; De Jesús y Menkes, 2014). ' Así, más allá de la inestabilidad en las parejas sexuales de los adolescentes, las evidencias empíricas han enfatizado en el importante rol que juegan las desigualdades de género y poder (que se intersectan e interactúan con otras desigualdades sociales como la clase social, la edad, etc.), la deserotización y el control sexual y reproductivo de las mujeres y la doble moral sexual prevaleciente en México sobre las prácticas sexuales y reproductivas de los y las adolescentes. Estas desigualdades dificultan las negociaciones en torno al uso de métodos anticonceptivos y condón en esta población, e incrementan los riesgos de contraer enfermedades de transmisión sexual así como la ocurrencia de embarazos no planeados (Sosa 2010; Warr, 2001; Gayet et al., 2003).

Paralelamente, las evidencias empíricas en relación al uso del condón sugieren que a pesar de un significativo incremento en su uso, es también evidente que no se ha logrado erotizar su uso ni erradicar los significados que lo asocian con la falta de amor y la desconfianza, así como con la incomodidad (Sosa, 2010), por lo que los significados (sociales y simbólicos) en torno al condón entran en conflicto con los discursos del amor, en los cuales se enfatiza la confianza y el compromiso mutuos, lo que es más, el uso del mismo tiende a limitarse cuando en la 'definición de la situación' se considera pertinente su uso, lo que generalmente tiende a vincularse con relaciones sexuales ocasionales, "no estables", cuando se conoce poco (o no se conoce) al compañero/a sexual (Szasz, 1998; Amuchástegui, 2007; Rodríguez, 2002). También se ha sugerido el importante rol de los condicionantes de género tanto en las definiciones sociales sobre el riesgo como en las negociaciones en torno a bajo qué circunstancias ocurren los encuentros sexuales (Sosa-Sánchez, 2010; De Jesús y Menkes, 2014).

A diferencia de otros métodos anticonceptivos, el uso de condón implica la necesidad de establecer negociaciones entre las personas involucradas, donde éstas puedan expresar bajo qué circunstancias desean que se

${ }^{1}$ Estos estudios sugieren que los varones también son objeto de condicionamientos sociales que los llevan a reproducir determinados atributos relacionados con estereotipos masculinos lo cual dificulta el establecimiento de intercambios más equitativos no sólo con las mujeres sino con otros varones. 
Jóvenes en México: ¿existen diferencias entre hombres y mujeres en su inicio sexual y uso del condón? / C. MENKES et al.

den los encuentros sexuales. Esto se torna problemático en el ámbito de la sexualidad, en contextos donde la maternidad, la ausencia de deseo y de experiencia erótico-sexual en las mujeres son altamente valorados y se convierten en atributo del ser mujer (Lamas, 2003; Giordano et al., 2009). Por el contrario, el mandato de experiencias eróticas y la búsqueda del placer se constituyen en unas de las principales formas de representación y reafirmación de la masculinidad (Aguirre y Güell, 2002; Giordano et al., 2009; Amuchástegui y Aggleton, 2007; Amuchástegui, 2007).

Sobre esta última se ha sugerido que los varones se enfrentan a presiones para tener múltiples parejas, a tener éxito con las mujeres para afirmar su virilidad, a no ser pasivos en las relaciones sexuales (Amuchástegui y Aggleton, 2007: 70). Estos estereotipos de sexualidad masculina y femenina repercuten en la valoración social diferenciada otorgada a la actividad sexual premarital y en un doble estándar sexual, donde la sexualidad femenina continua estando dirigida hacia fines esencialmente reproductivos (Pick, Givaudan y Kline, 2005; Jones, 2010), donde la moral judeocristiana (y la religión católica) juegan un rol central.

El nivel de instrucción es otro factor que ha sido señalado como importante en el inicio de la actividad sexual del adolescente. Oliva, Serra y Vallejo (1997) indican que el nivel educativo propio y el de los padres influyen en los comportamientos sexuales de las y los adolescentes. De tal suerte, un mayor nivel educativo se relaciona con un inicio más tardío de las relaciones sexuales, con mayor vinculación de la afectividad en la sexualidad y con compartir con más frecuencia la iniciativa con la pareja en las relaciones coitales (De Jesús y Menkes, 2016). Así, las evidencias sugieren que el nivel de instrucción puede tender a igualar los comportamientos sexuales en ambos sexos.

Sin embargo existe un comportamiento sexual heterogéneo según los distintos contextos o regiones. América Latina se caracteriza por un inicio sexual en edades más tempranas que otras regiones del desarrollo como Asia, pero mucho más tardío que África. Dentro de América Latina, los datos para México muestran que este país se encuentra entre los de menor proporción de sexualmente activos, sobre todo por el comportamiento de las mujeres (Gayet et al., 2003).

En México, a pesar de que se plantea que por la influencia de la revolución sexual estadunidense, los jóvenes inician su vida sexual a edades cada vez más tempranas, varios autores han encontrado que la edad de inicio sexual se ha pospuesto, utilizando indicadores robustos tal como la proporción de las sexualmente activas a una edad exacta, tablas de vida 
y modelos de regresión de Cox (Gayet y Solís, 2007; Welti, 2005; Palma y González, 2010, Menkes y Serrano, 2010). En suma, podemos afirmar que la relación entre la salud sexual y reproductiva, los comportamientos y prácticas relativas a éstas últimas y las desigualdades de género son muy complejas y una encuesta cuantitativa únicamente puede reflejar algunas tendencias generales.

Es indudable que la salud sexual y reproductiva se relaciona con la diversidad de situaciones de la población, contextos, coyunturas, eventos de vida, valores, moral sexual y preferencias personales de los jóvenes y que se sugiere cautela al buscar generalidades (Stern y Menkes, 2012); pero no se puede soslayar tampoco el hecho de que se necesitan más estudios que revisen la relación entre la reproducción las prácticas sexuales, la sexualidad, las desigualdades de género y las prácticas de riesgo. Por tanto el objetivo de este documento, es analizar las características del inicio sexual de los jóvenes mexicanos de 12 a 29 años de edad, así como los factores que se relacionan con el uso del condón, teniendo como eje conceptual la perspectiva de género y enfatizando en el rol de las desigualdades de género sobre las prácticas sexuales en esta población. Para ello se analizan en primer lugar, las prácticas sexuales de los jóvenes en distintos contextos, comparando entre hombres y mujeres. En la segunda parte del trabajo se estudia el uso de métodos anticonceptivos de los jóvenes en la primera relación sexual.

Nuestra principal pregunta de investigación consiste en saber si existen diferencias sociodemográficas entre hombres y mujeres en lo que respecta a su inicio sexual y al uso del preservativo en esa primera relación sexual. Asimismo, nos preguntamos cómo influyen los estereotipos de género en ambos eventos. Partimos de la hipótesis de que los estereotipos de género tradicionales obstaculizan la protección sexual en los diferentes contextos sociales ya que las expectativas asociadas a los roles de género (que se expresan a través de los estereotipos de género) condicionan las negociaciones que tienen lugar durante el debut sexual y sobre el uso del preservativo.

\section{Metodología}

El presente es un estudio cuantitativo de corte transversal, descriptivo-correlacional. La población de estudio fueron mujeres y varones de 12 a 29 años. La fuente de datos utilizada fue la Encuesta Nacional de la Juventud 2010 (ENJ 2010), que es una encuesta que cuenta con datos representativos a nivel nacional, estatal y para seis zonas metropolitanas, la cual tiene un diseño de muestra probabilístico, polietápico, estratificado y por con- 
Jóvenes en México: ¿existen diferencias entre hombres y mujeres en su inicio sexual y uso del condón? / C. MENKES et al.

glomerados, contando con una muestra nacional de 29,787 cuestionarios individuales.

Para estudiar las características del debut sexual se establecen análisis bi-variados que nos permitan analizar la asociación entre las características socio-demográficas de los jóvenes con la edad de inicio sexual y la de su pareja. Además se estiman dos modelos de regresión logística, incluyendo como variable dependiente si se iniciaron o no en la adolescencia y las variables independientes comprenden el estrato socio-económico del hogar ${ }^{2}$, la condición de habla indígena, y el índice de estereotipos de género. En el primer modelo el universo de estudio está constituido únicamente por las mujeres de 20 a 29 años y en el segundo modelo los varones también de 20 a 29 años. Cabe aclarar que en estas estimaciones únicamente incluimos a los jóvenes no adolescentes mayores de 19 años, pues de lo contrario no podríamos saber si en el futuro iban a tener relaciones sexuales aún siendo adolescentes.

Paralelamente, con el fin de conocer la probabilidad de asociación de factores sociodemográficos con una mejor protección sexual, se construyeron dos modelos de regresión logística, obteniendo razones de momios (RM) con intervalos de confianza de 95 por ciento (IC 95 por ciento). La variable dependiente se construyó tomando en cuenta si los jóvenes de 12 a 29 años o sus parejas usaron o no un preservativo masculino o femenino en la primera relación sexual, ${ }^{3}$ mientras que las variables explicativas fueron el estrato socioeconómico del hogar, la condición de habla indígena, el tamaño de localidad, la generación de pertenencia, el índice de estereotipos de género ${ }^{4}$, con quién tuvo su primera relación sexual, además de la edad de inicio sexual y la edad de la pareja sexual. El universo de estudio lo conforman en la primera regresión las mujeres y en la segunda los varones de 12 a 29 años sexualmente activos.

El índice de estereotipos de género se construyó tomando en cuenta las siguientes 20 preguntas de la encuesta de la ENJ 2010, presentadas en la Tabla 1.

\footnotetext{
${ }^{2}$ La construcción de los estratos socioeconómicos del hogar fueron construidos por Carlos Echarri (Echarri, 2008; Echarri, 2016) tomando en cuenta la escolaridad de todos los miembros del hogar, la ocupación y las características de la vivienda.

${ }^{3}$ Se analiza únicamente el uso del condón ya que es el único método que protege de un embarazo y/o de las infecciones de transmisión sexual.

${ }^{4}$ Vale la pena señalar que la información recabada sólo incluye a hombres y mujeres por lo que no es posible incluir otro género en el análisis.
} 
Tabla 1: Preguntas introducidas en el Índice de Género

Te voy a leer algunas preguntas, dime ¿qué tan de acuerdo estás con ellas?: 1= Totalmente de acuerdo, 2 = De acuerdo, $3=\mathrm{Ni}$ de acuerdo ni en desacuerdo, $4=$ En desacuerdo $5=$ Totalmente en desacuerdo

\begin{tabular}{|c|c|c|}
\hline Preguntas & Respuesta & $\begin{array}{l}\text { Estereotipos } \\
\text { de género }\end{array}$ \\
\hline \multirow{3}{*}{$\begin{array}{l}\text { 1. ¿Estás de acuerdo en que la función más importante de la mujer es ser } \\
\text { madre? }\end{array}$} & 1 y 2 & Tradicional \\
\hline & 3,4 y 5 & No tradicional \\
\hline & 3,4 y 5 & No tradicional \\
\hline \multirow{2}{*}{$\begin{array}{l}\text { 2. ¿Estás de acuerdo, en que cuando una mujer hace algo indebido el } \\
\text { hombre tiene derecho a pegarle? }\end{array}$} & 1 y 2 & Tradicional \\
\hline & 3,4 y 5 & No tradicional \\
\hline \multirow{2}{*}{$\begin{array}{l}\text { 3. ¿Estás de acuerdo en que el hombre siempre tiene la obligación de } \\
\text { mantener a su familia? }\end{array}$} & 1 y 2 & Tradicional \\
\hline & 3,4 y 5 & No tradicional \\
\hline \multirow{3}{*}{$\begin{array}{l}\text { 4. ¿Estás de acuerdo en que una mujer tiene derecho a decidir cuántos } \\
\text { hijos tener y cuándo tenerlos? }\end{array}$} & 3,4 y 5 & Tradicional \\
\hline & 3,4 y 5 & Tradicional \\
\hline & 1 y 2 & No tradicional \\
\hline \multirow{2}{*}{ 5. ¿Estás de acuerdo en que está bien que una pareja decida no tener hijos? } & 3,4 y 5 & Tradicional \\
\hline & 1 y 2 & No tradicional \\
\hline \multirow{2}{*}{$\begin{array}{l}\text { 6. ¿Estás de acuerdo que aunque las mujeres no quieran, es su obligación } \\
\text { tener relaciones sexuales con sus parejas? }\end{array}$} & 1 y 2 & Tradicional \\
\hline & 3,4 y 5 & No tradicional \\
\hline \multirow{2}{*}{ 7. ¿Estás de acuerdo, en que el hombre es más agresivo que la mujer? } & 1 y 2 & Tradicional \\
\hline & 3,4 y 5 & No tradicional \\
\hline \multirow{2}{*}{$\begin{array}{l}\text { 8. ¿Estás de acuerdo, en que las mujeres no pueden desempeñar las mismas } \\
\text { actividades que los hombres? }\end{array}$} & 1 y 2 & Tradicional \\
\hline & 3,4 y 5 & No tradicional \\
\hline \multirow{2}{*}{ 9. ¿Estás de acuerdo, en que el hombre piensa más las cosas que la mujer? } & 1 y 2 & Tradicional \\
\hline & 3,4 y 5 & No tradicional \\
\hline \multirow{2}{*}{$\begin{array}{l}\text { 10. ¿Estás de acuerdo, en que un hombre, a diferencia de una mujer, } \\
\text { necesita varias parejas sexuales? }\end{array}$} & 1 y 2 & Tradicional \\
\hline & 3,4 y 5 & No tradicional \\
\hline \multirow{2}{*}{$\begin{array}{l}\text { 11. ¿Estás de acuerdo en que un verdadero hombre no debe mostrar sus } \\
\text { debilidades y sentimientos? }\end{array}$} & 1 y 2 & Tradicional \\
\hline & 3,4 y 5 & No tradicional \\
\hline \multirow{2}{*}{ 12. ¿Estás de acuerdo en que la mujer debe de llegar virgen al matrimonio? } & 1 y 2 & Tradicional \\
\hline & 3,4 y 5 & No tradicional \\
\hline \multirow{2}{*}{ 13. ¿Estás de acuerdo, en que el hombre es infiel por naturaleza? } & 1 y 2 & Tradicional \\
\hline & 3,4 y 5 & No tradicional \\
\hline \multirow{2}{*}{$\begin{array}{l}\text { 14. ¿Estás de acuerdo en que la vida es más dura para el hombre que para } \\
\text { la mujer? }\end{array}$} & 1 y 2 & Tradicional \\
\hline & 3,4 y 5 & No tradicional \\
\hline \multirow{2}{*}{$\begin{array}{l}\text { 15. ¿Estás de acuerdo, en que la mujer tiene mayor capacidad para cuidar a } \\
\text { los hijos enfermos? }\end{array}$} & 1 y 2 & Tradicional \\
\hline & 3,4 y 5 & No tradicional \\
\hline \multirow{2}{*}{$\begin{array}{l}\text { 16. ¿Estás de acuerdo en que una buena esposa debe dedicarse } \\
\text { exclusivamente al hogar y al cuidado del marido? }\end{array}$} & 1 y 2 & Tradicional \\
\hline & 3,4 y 5 & No tradicional \\
\hline \multirow{2}{*}{$\begin{array}{l}\text { 17. ¿Estás de acuerdo, en que los hijos son mejor educados por una madre } \\
\text { que por un padre? }\end{array}$} & 1 y 2 & Tradicional \\
\hline & 3,4 y 5 & No tradicional \\
\hline \multirow{2}{*}{$\begin{array}{l}\text { 18. ¿Estás de acuerdo, en que aunque las mujeres trabajen fuera de la casa, } \\
\text { es el hombre el que debe hacerse responsable del sostén de la familia? }\end{array}$} & $1 \mathrm{y} 2$ & Tradicional \\
\hline & 3,4 y 5 & No tradicional \\
\hline \multirow{2}{*}{$\begin{array}{l}\text { 19. ¿Estás de acuerdo en que los hijos hacen más caso cuando les llama la } \\
\text { atención el padre, que la madre? }\end{array}$} & 1 y 2 & Tradicional \\
\hline & 3,4 y 5 & No tradicional \\
\hline \multirow{2}{*}{$\begin{array}{l}\text { 20. ¿Estás de acuerdo en que un buen hombre es el que provee } \\
\text { económicamente a su familia? }\end{array}$} & 1 y 2 & Tradicional \\
\hline & 3,4 y 5 & No tradicional \\
\hline
\end{tabular}

Fuente: elaboración propia con datos de la Encuesta Nacional de la Juventud 2010. 
Jóvenes en México: ¿existen diferencias entre hombres y mujeres en su inicio sexual y uso del condón? / C. MENKES et al.

Se agruparon las respuestas del índice de género de la siguiente forma: Cuando los estudiantes estuvieron de acuerdo o totalmente de acuerdo en las respuestas $1,2,3,6,7,8,9,10,11,12,13,14,15,16,17,18,19$ y 20 se consideraron tradicionales y los que contestaron ni de acuerdo ni en desacuerdo, no estoy de acuerdo, o totalmente en desacuerdo, como no tradicionales. En cambio en los rubros 4 y 5 cuando los estudiantes contestaron estar de acuerdo o totalmente de acuerdo, se consideraron como respuestas no tradicionales y el resto como tradicionales.

Vale la pena señalar que en la medida en que este índice no ha sido probado en otras investigaciones, se consideró importante calcular el alpha de Cronbach $^{5}$ para probar su consistencia estadística y el resultado del valor del alpha de Cronbach fue de 0.87 , valor que hace del índice de género un índice estadísticamente altamente consistente.

El valor de cada variable fue 0 cuando se mostró una actitud tradicional y 1 una actitud más moderna. A cada adolescente se le asignó un valor sumando el resultado de los rubros, si el valor resultó ser de 0 a 12 se consideró tradicional y de 12 a 20 no tradicional. Para esto, se tomó en cuenta el porcentaje más cercano a 50 por ciento de los casos para poder dividir la variable en dos categorías tomando en cuenta también el grado de correlación entre las variables.

\section{RESUltados}

\section{Iniciación sexual}

En la Tabla 2 se observa que prácticamente uno de cada dos jóvenes de 12 a 29 años se ha iniciado sexualmente. Como tradicionalmente ha sucedido en México, un número mayor de varones que de mujeres reporta haber tenido al menos una relación sexual (54vs. 49 por ciento respectivamente). Además es muy claro, según los datos, que los varones se inician sexualmente a una edad menor que las mujeres: mientras que 38.3 por ciento de los hombres de la generación 1991-1995 (que en el momento de la encuesta tenían entre 15 a 19 años) ya se había iniciado sexualmente, únicamente 28.8 por ciento de las mujeres de esa misma generación ya había tenido relaciones sexuales.

\footnotetext{
${ }^{5}$ El alpha de Cronbach es una medida de consistencia interna del índice que expresa el grado en que los ítems miden el mismo fenómeno.
} 
Tabla 2: Porcentaje de jóvenes de 12 a 29 años que han tenido relaciones sexuales por generación y sexo

\begin{tabular}{lrrr}
\hline \multicolumn{2}{c}{ Sexo } & Mujer & Total \\
\hline $1996-1998$ & Hombre & 2.5 & 2.3 \\
$1991-1995$ & 2.10 & 28.8 & 33.6 \\
$1990-1986$ & 38.3 & 71.1 & 75.7 \\
$1981-1985$ & 80.9 & 86.5 & 89.0 \\
Total & 91.7 & 49.0 & 51.4 \\
\hline Fuente: elaboración propia con datos de la Encuesta Nacional de la Juventud 2010.
\end{tabular}

En cuanto a la distribución por edad, se constata que la edad de inicio sexual se concentra en el grupo de 15 a 19 años, tanto en el caso de los hombres como en el de las mujeres (55.1 y 45.2 por ciento, respectivamente). La edad de la pareja sexual también se concentra en el grupo de 15 a 19 años (51.9 por ciento) en el caso de los varones, mientras que en el sexo femenino se concentra en el grupo de 25 a 29 años (48.3 por ciento) (Ver Tabla 3). Como se aprecia, los resultados de la encuesta refuerzan los hallazgos de la literatura sobre el tema en la que se postula que, por los mandatos y normatividades de género, los hombres inician su vida sexual antes que las mujeres y, éstas últimas, se inician con parejas de mayor edad.

Tabla 3: Distribución porcentual de la edad a la primera relación sexual y la edad de la pareja sexual de los jovenes de 12 a 29 años por sexo

\begin{tabular}{lrrrr}
\hline & \multicolumn{2}{c}{ Hombre } & \multicolumn{2}{c}{ Mujer } \\
\cline { 2 - 5 } $\begin{array}{l}\text { Edad de } \\
\text { inicio sexual }\end{array}$ & $\begin{array}{r}\text { Edad de la } \\
\text { 1ralación } \\
\text { sexual }\end{array}$ & $\begin{array}{r}\text { Edad de la } \\
\text { pareja sexual }\end{array}$ & $\begin{array}{r}\text { Edad de la } \\
\text { rad relación } \\
\text { sexual }\end{array}$ & $\begin{array}{r}\text { Edad de la } \\
\text { pareja sexual }\end{array}$ \\
\hline 12-14 años & 14.5 & 11.3 & 8.0 & 1.7 \\
15 a 19 años & 55.1 & 51.9 & 45.2 & 21.2 \\
20 a 24 años & 21.2 & 22.4 & 28.3 & 28.8 \\
25 a 29 años & 9.1 & 14.4 & 18.6 & 48.3 \\
Total & 100.0 & 100.0 & 100.0 & 100.0 \\
Media & 16.6 & 17.4 & 17.5 & 20.5 \\
Mediana & 17.0 & 17.0 & 17.0 & 20.0 \\
\hline Fuente: elaboración propia con datos de la Encuesta Nacional de la Juventud 2010.
\end{tabular}


Jóvenes en México: ¿existen diferencias entre hombres y mujeres en su inicio sexual y uso del condón? / C. MENKES et al.

Ante la pregunta de cuál fue la razón principal del inicio sexual, se observa en la Tabla 4 que 46.7 por ciento respondió que por amor, 22.5 por ciento por curiosidad, 17.5 por ciento porque lo quisieron, y 6.1 por ciento porque no pudieron controlarse. Únicamente 3.7 por ciento de todos los jóvenes lo hizo porque se casó. Si se compara por sexo, se observan importantes diferencias en las razones que dieron los jóvenes con respecto a su inicio sexual: 62.2 por ciento de las mujeres considera que se inició sexualmente por amor y únicamente 32.1 por ciento de los hombres. De hecho, la principal razón en los varones es que se iniciaron por curiosidad y en las mujeres, por amor. Sin duda alguna, la idea del amor romántico sigue siendo una justificación válida para el inicio sexual en el sexo femenino, mientras que para los varones no ocurre ello, pues un mayor número de varones declaró que se inició porque quiso (20.9 por ciento) y porque no pudo controlarse (8.9 por ciento).

Tabla 4: Distribución porcentual de las razones por las cuales los jóvenes de 12 a 29 años tuvieron su primera relación sexual según sexo

\begin{tabular}{lrrr}
\hline & \multicolumn{2}{c}{ Sexo } & \\
Razón para tener su 1ra. relación sexual & Hombre & Mujer & Total \\
\hline Por amor & 32.1 & 62.2 & 46.7 \\
Por curiosidad & 33.8 & 10.5 & 22.5 \\
Porque quise (voluntad propia) & 20.9 & 13.9 & 17.5 \\
Porque no pude controlarme & 8.9 & 3.1 & 6.1 \\
Porque mi pareja (novio (a)) me convenció & 2.0 & 2.9 & 2.4 \\
Porque me casé o uní & 1.2 & 6.5 & 3.7 \\
Porque me obligaron & 0.3 & 0.6 & 0.4 \\
Otra & 0.9 & 0.3 & 0.6 \\
Total & 100.0 & 100.0 & 100.0 \\
\hline
\end{tabular}

Fuente: elaboración propia con datos de la Encuesta Nacional de la Juventud 2010.

Estos resultados se confirman si analizamos la relación con la persona con la que tuvieron su primera relación sexual según la Tabla 5, pues si bien la mayoría de los jóvenes se inició con el novio o la novia, casi 30 por ciento de los varones se inició con su amiga, mientras que las mujeres cerca de siete por ciento fue con un amigo. Por el contrario, 7.8 por ciento de los varones se inició con la esposa y 35.4 por ciento de las mujeres tuvieron su primera relación sexual con su marido. 
Tabla 5: Distribución porcentual de jóvenes iniciados sexualmente según persona con quien tuvieron su primera relación sexual

\begin{tabular}{lrrr}
\hline Tipo de pareja en la & Sexo & & \\
1ra Relación sexual & Hombre & Mujer & Total \\
\hline Amigo(a) & 29.9 & 6.9 & 18.7 \\
Novio(a) & 57.3 & 57.1 & 57.2 \\
Esposo(a) & 7.8 & 35.4 & 21.2 \\
Sexo servidor(a) & 3.6 & 0.1 & 1.9 \\
Un familiar & 1.0 & 0.2 & 0.6 \\
Otra & 0.4 & 0.4 & 0.4 \\
Total & 100.0 & 100.0 & 100.0 \\
\hline
\end{tabular}

Fuente: elaboración propia con datos de la Encuesta Nacional de la Juventud 2010.

Según la Tabla 6, al preguntarles a los jóvenes no iniciados sexualmente por qué no han tenido relaciones sexuales, el mayor porcentaje contestó que no consideran tener edad suficiente (34.4 por ciento). Sin embargo, las diferencias por sexo se manifiestan también sobre todo porque un porcentaje mayor de mujeres que de hombres declaró querer llegar virgen al matrimonio (10.7 vs. dos por ciento) y por el contrario, un mayor número de varones que no han tenido relaciones sexuales declara que es porque no han tenido la oportunidad, lo que puede significar que lo viven como un evento que si se presenta, se debe aprovechar (11.6 vs. 2.6 por ciento).

Tabla 6: Distribución porcentual de las razones por las cuáles los jóvenes no han tenido relaciones sexuales por sexo

\begin{tabular}{lrrr}
\hline & \multicolumn{2}{c}{ Sexo } & \\
\cline { 2 - 3 } Razón por la cual no han tenido & & & \\
relaciones sexuales & Hombre & Mujer & Total \\
\hline Por miedo al SIDA o a infecciones & 6.9 & 4.2 & 5.5 \\
de transmisión sexual & 7.4 & 9.2 & 8.3 \\
Por miedo al embarazo & 11.6 & 2.6 & 6.8 \\
Porque no he tenido oportunidad & 2.0 & 10.7 & 6.7 \\
Porque quiero llegar virgen al matrimonio & 9.7 & 10.1 & 9.9 \\
Espero a la pareja ideal & 37.0 & 32.2 & 34.4 \\
Porque no tengo edad suficiente & 21.9 & 27.6 & 25.0 \\
No quiero & 1.0 & .9 & 1.0 \\
Mi religión no me lo permite & 2.4 & 2.5 & 2.5 \\
Otra & $J u v$
\end{tabular}

Fuente: elaboración propia con datos de la Encuesta Nacional de la Juventud 2010. 
Jóvenes en México: ¿existen diferencias entre hombres y mujeres en su inicio sexual y uso del condón? / C. MENKES et al.

Si se observa la Tabla 7, la distribución por edad en la primera relación sexual de acuerdo al estrato socioeconómico del hogar, se puede ver que en el caso de los varones no existe una relación clara en el inicio sexual según esta variable. Únicamente se ve un porcentaje mayor de inicio sexual entre los 20 y 29 años en el estrato Muy bajo, tanto del iniciado como de la pareja sexual. Por otro lado, llama la atención el elevado porcentaje de jóvenes varones iniciados sexualmente entre los 12 y los 14 años (14.5 por ciento); este porcentaje disminuye en el caso de la pareja sexual (11.2 por ciento). Con respecto al sexo femenino, se observa claramente una disminución del porcentaje de iniciadas sexualmente entre 12 y 14 años a medida en que crece el estrato socioeconómico. En cuanto a la pareja sexual de las mujeres, llama la atención que en todos los estratos socioeconómicos, cerca de la mitad de las mujeres se inició con una pareja de 20 años o más.

En lo que concierne a los que declararon hablar una lengua indígena se constata, en el caso de los varones, un mayor porcentaje de iniciados sexualmente después de los 20 años con respecto a los no indígenas mientras que en el caso de las mujeres indígenas frente a las mujeres que no hablan una lengua indígena, la diferencia más sobresaliente consiste en el porcentaje tan elevado de iniciadas sexualmente entre los 12 a 14 años de edad (14.9 por ciento) en el caso de las hablantes de lengua indígena mientras que, entre las mujeres jóvenes no hablantes de lengua indígena, únicamente 7.7 por ciento se iniciaron sexualmente a edades tan tempranas, lo que puede estar indicando un inicio sexual más temprano asociado a contextos y expectativas culturales en relación a las prácticas sexuales y reproductivas, asociados no sólo con el género sino con la afiliación étnico-racial.

En cuanto al tamaño de localidad, se puede ver que el mayor porcentaje de varones iniciados sexualmente entre los 12 y los 14 años corresponde a las zonas urbanas y por el contrario, se observa un menor porcentaje de varones que se iniciaron después de los 20 años en comparación con las zonas rurales. En el caso de las mujeres se observan las mismas tendencias. Con respecto a la pareja sexual, en ambos sexos existe un mayor porcentaje de parejas mayores de 19 años en las zonas rurales. Si se analiza la relación entre el inicio sexual y los estereotipos de género, llama la atención que los que expresaron opiniones más tradicionales presentan un mayor porcentaje de inicio sexual de 12 a 14 años, que los que mostraron tener opiniones menos tradicionales respecto a los roles de género. Un ejemplo de ello es que 10.1 por ciento de las mujeres con opiniones tradicionales se iniciaron sexualmente entre los 12 y 14 años, mientras que para las no tradicionales este porcentaje corresponde a 6.9 por ciento. La misma tendencia se observa en la edad de la pareja sexual en ambos sexos. 
Tabla 7: Edad de la primera relación sexual en jóvenes mexicanos según características sociodemográficas (\%)

\begin{tabular}{|l|r|r|r|r|r|r|r|r|r|r|}
\hline \multicolumn{9}{|c|}{ Sexo masculino } & \multicolumn{7}{|c|}{ Pareja del sexo masculino } \\
\hline Estratos socioeconómicos \\
\hline $\begin{array}{l}\text { Edad de la 1ra } \\
\text { relación sexual }\end{array}$ & $\begin{array}{r}\text { Muy } \\
\text { bajo }\end{array}$ & Bajo & Medio & Alto & Total & $\begin{array}{r}\text { Muy } \\
\text { bajo }\end{array}$ & Bajo & Medio & Alto & Total \\
\hline 12 a 14 años & 15.2 & 16.1 & 14.0 & 13.3 & 14.5 & 13.1 & 13.4 & 10.2 & 9.2 & 11.2 \\
\hline 15 a 19 años & 72.8 & 75.5 & 76.7 & 78.3 & 76.3 & 66.4 & 71.3 & 76.0 & 75.8 & 73.4 \\
\hline 20 a 29 años & 12.0 & 8.4 & 9.3 & 8.5 & 9.1 & 20.5 & 15.3 & 13.8 & 15.0 & 15.4 \\
\hline Total & 100.0 & 100.0 & 100.0 & 100.0 & 100.0 & 100.0 & 100.0 & 100.0 & 100.0 & 100.0 \\
\hline
\end{tabular}

\begin{tabular}{|l|r|r|r|r|r|r|}
\hline Hablantes de lengua indigena \\
\hline $\begin{array}{l}\text { Edad de la 1ra } \\
\text { relación sexual }\end{array}$ & Indígena & No Indígena & Total & Indígena & No Indígena & Total \\
\hline 12 a 14 años & 13.8 & 14.6 & 14.5 & 9.6 & 11.2 & 11.2 \\
\hline 15 a 19 años & 72.6 & 76.5 & 76.4 & 71.2 & 73.6 & 73.5 \\
\hline 20 a 29 años & 13.6 & 8.9 & 9.1 & 19.2 & 15.2 & 15.4 \\
\hline Total & 100.0 & 100.0 & 100.00 & 100.0 & 100.0 & 100.00 \\
\hline
\end{tabular}

\begin{tabular}{|l|r|r|r|r|r|r|r|r|}
\hline \multicolumn{2}{|l|}{ Tipo de localidad } & \multicolumn{1}{|c|}{} & & & & & \\
\hline $\begin{array}{l}\text { Edad de la 1ra } \\
\text { relación sexual }\end{array}$ & Urbana & No Urbana & Rural & Total & Urbana & No Urbana & Rural & Total \\
\hline 12 a 14 años & 15.4 & 13.9 & 11.8 & 14.5 & 11.4 & 10.7 & 10.6 & 11.2 \\
\hline 15 a 19 años & 76.6 & 74.8 & 76.4 & 76.4 & 73.8 & 72.0 & 73.2 & 73.5 \\
\hline 20 a 29 años & 8.0 & 11.3 & 11.8 & 9.1 & 14.8 & 17.3 & 16.2 & 15.4 \\
\hline Total & 100.0 & 100.0 & 100.0 & 100.0 & 100.0 & 100.0 & 100.0 & 100.0 \\
\hline
\end{tabular}

\begin{tabular}{|l|r|r|r|r|r|r|}
\hline Índice de género & & & & \\
\hline $\begin{array}{l}\text { Edad de la 1ra } \\
\text { relación sexual }\end{array}$ & Tradicional & No tradicional & Total & Tradicional & No tradicional & Total \\
\hline 12 a 14 años & 15.6 & 13.5 & 14.4 & 12.5 & 10.3 & 11.2 \\
\hline 15 a 19 años & 75.2 & 77.2 & 76.4 & 71.4 & 75.1 & 73.5 \\
\hline 20 a 29 años & 9.2 & 9.3 & 9.2 & 16.2 & 14.7 & 15.3 \\
\hline
\end{tabular}

\begin{tabular}{|l|r|r|r|r|r|r|r|r|r|r|}
\hline Generación de pertenencia \\
\hline Edad de la 1ra & 1996 & 1991 & 1990 & 1981 & & 1996 & 1991 & 1990 & 1981 & \\
relación sexual & 1998 & 1995 & 1986 & 1985 & Total & 1998 & 1995 & 1986 & 1985 & Total \\
\hline 12 a 14 años & 100.0 & 24.3 & 11.5 & 9.4 & 14.5 & 62.3 & 18.7 & 8.7 & 7.6 & 11.2 \\
\hline 15 a 19 años & & 75.7 & 80.9 & 73.5 & 76.4 & 34.4 & 75.5 & 75.9 & 70.2 & 73.5 \\
\hline 20 a 29 años & & & 7.6 & 17.1 & 9.1 & 3.3 & 5.9 & 15.4 & 22.2 & 15.4 \\
\hline Total & 100.0 & 100.0 & 100.0 & 100.0 & 100.0 & 100.0 & 100.0 & 100.0 & 100.0 & 100.0 \\
\hline
\end{tabular}

Fuente: elaboración propia con datos de la Encuesta Nacional de la Juventud 2010. 
Jóvenes en México: ¿existen diferencias entre hombres y mujeres en su inicio sexual y uso del condón? / C. MENKES et al.

Tabla 7 continuación: Edad de la primera relación sexual en jóvenes mexicanos según características sociodemográficas (\%)

\begin{tabular}{|l|r|r|r|r|r|r|r|r|r|r|}
\hline \multicolumn{9}{|c|}{ Sexo femenino } & \multicolumn{7}{|c|}{ Pareja del sexo femenino } \\
\hline Estratos socioeconómicos & $\begin{array}{r}\text { Muy } \\
\text { bajo }\end{array}$ & Bajo & Medio & Alto & Total & $\begin{array}{r}\text { Muy } \\
\text { bajo }\end{array}$ & Bajo & Medio & Alto & Total \\
\hline $\begin{array}{l}\text { Edad de la 1ra } \\
\text { relación sexual }\end{array}$ & 12.1 & 9.0 & 7.3 & 4.8 & 8.0 & 2.1 & 2.2 & 1.2 & 1.0 & 1.6 \\
\hline 12 a 14 años & 11.9 & 77.2 & 71.1 & 71.7 & 73.4 & 46.5 & 50.0 & 49.6 & 45.5 & 48.3 \\
\hline 15 a 19 años & 16.0 & 13.8 & 21.7 & 23.5 & 18.6 & 51.5 & 47.8 & 49.1 & 53.5 & 50.0 \\
\hline 20 a 29 años & 100.0 & 100.0 & 100.0 & 100.0 & 100.0 & 100.0 & 100.0 & 100.0 & 100.0 & 100.0 \\
\hline Total &
\end{tabular}

\begin{tabular}{|l|r|r|r|r|r|r|}
\hline \multicolumn{7}{|l|}{ Hablantes de lengua indigena } \\
\hline $\begin{array}{l}\text { Edad de la 1ra } \\
\text { relación sexual }\end{array}$ & Indígena & No Indígena & Total & Indígena & No Indígena & Total \\
\hline 12 a 14 años & 14.5 & 7.7 & 8.0 & 2.5 & 1.6 & 1.6 \\
\hline 15 a 19 años & 71.3 & 73.5 & 73.5 & 45.5 & 48.4 & 48.3 \\
\hline 20 a 29 años & 14.2 & 18.8 & 18.6 & 50.2 & 50.0 & 50.0 \\
\hline Total & 100.0 & 100.0 & 100.0 & 100.0 & 100.0 & 100.0 \\
\hline
\end{tabular}

\begin{tabular}{|l|r|r|r|r|r|r|r|r|}
\hline Tipo de localidad & \multicolumn{10}{|l|}{} & & & \\
\hline $\begin{array}{l}\text { Edad de la 1ra } \\
\text { relación sexual }\end{array}$ & Urbana & No Urbana & Rural & Total & Urbana & No Urbana & Rural & Total \\
\hline 12 a 14 años & 8.3 & 6.6 & 7.8 & 8.0 & 1.8 & 9.0 & 1.4 & 1.6 \\
\hline 15 a 19 años & 74.1 & 72.9 & 71.5 & 73.4 & 49.9 & 47.2 & 43.9 & 48.3 \\
\hline 20 a 29 años & 17.6 & 20.5 & 20.7 & 18.6 & 48.3 & 51.9 & 54.7 & 50.0 \\
\hline Total & 100.0 & 100.0 & 100.0 & 100.0 & 100.0 & 100.0 & 100.0 & 100.0 \\
\hline
\end{tabular}

\begin{tabular}{|l|r|r|r|r|r|r|}
\hline Índice de género & & & & & \\
\hline $\begin{array}{l}\text { Edad de la 1ra } \\
\text { relación sexual }\end{array}$ & Tradicional & No tradicional & Total & Tradicional & No tradicional & Total \\
\hline 12 a 14 años & 10.1 & 6.9 & 8.2 & 2.0 & 1.5 & 1.7 \\
\hline 15 a 19 años & 74.4 & 77.5 & 73.2 & 46.1 & 49.6 & 48.3 \\
\hline 20 a 29 años & 15.4 & 20.6 & 18.6 & 51.8 & 48.9 & 50.0 \\
\hline
\end{tabular}

\begin{tabular}{|c|c|c|c|c|c|c|c|c|c|c|}
\hline \multicolumn{11}{|c|}{ Generación de pertenencia } \\
\hline Edad de la 1ra & 1996 & 1991 & 1990 & 1981 & & 1996 & 1991 & 1990 & 1981 & \\
\hline relación sexual & 1998 & 1995 & 1986 & 1985 & Total & 1998 & 1995 & 1986 & 1985 & Total \\
\hline 12 a 14 años & 100.0 & 15.5 & 6.8 & 3.8 & 8.0 & 31.4 & 2.4 & 1.7 & 0.6 & 1.6 \\
\hline 15 a 19 años & & 84.5 & 78.6 & 64.7 & $\overline{73.4}$ & 50.7 & 68.4 & 49.2 & 37.6 & 48.3 \\
\hline 20 a 29 años & & & 14.7 & 31.5 & 18.6 & 17.9 & 29.2 & 49.1 & 61.8 & 50.0 \\
\hline Total & 100.0 & 100.0 & 100.0 & 100.0 & 100.0 & 100.0 & 100.0 & 100.0 & 100.0 & 100.0 \\
\hline
\end{tabular}

Fuente: elaboración propia con datos de la Encuesta Nacional de la Juventud 2010. 
Finalmente en lo que respecta a la generación de pertenencia, hay evidencia en la misma tabla de que en todas las generaciones existe un mayor porcentaje de varones iniciados sexualmente de 12 a 14 años que de mujeres; en cambio, existe un mayor porcentaje de mujeres iniciadas de los 20 a 29 años. Lo mismo sucede con la pareja sexual.

A modo de resumen, en lo que concierne a las características del inicio sexual, los resultados sugieren que las diferencias entre hombres y mujeres son muy claras: los varones se inician antes que las mujeres y éstas se inician con parejas de mayor edad (lo que podría estar asociado a una erotización de las diferencias de edad configurada socialmente y en donde la expectativa social indica que las mujeres deben tener parejas sexuales (hombres) de mayor edad. La única excepción se encuentra entre los no hablantes de lengua indígena donde un porcentaje ligeramente mayor de niñas que de niños se inició de 12 a 14 años aunque, al igual que en el resto de todas las mujeres, tienden a iniciarse con parejas mucho más grandes que ellas.

Otro dato que vale la pena subrayar, se refiere a que el estrato socioeconómico del hogar muestra una relación con la edad de inicio sexual del sexo femenino, ya que en la medida en que mejora el estrato socioeconómico, se retrasa la edad de inicio sexual; en cambio, no se observa una relación clara en el caso de los varones. También resulta importante recalcar que las características de la pareja sexual son diferentes según el sexo: con mayor frecuencia las mujeres se inician sexualmente con el novio y por amor, mientras que un porcentaje más elevado de hombres que de mujeres se inicia con una amiga, por curiosidad. Asimismo, las mujeres se inician, en general, con parejas más grandes que ellas.

\section{FACTORES ASOCIADOS CON HABERSE INICIADO SEXUALMENTE EN LA ADOLESCENCIA}

Vale recordar que desde el punto de vista demográfico, el inicio sexual temprano expone por más tiempo a los jóvenes al riesgo de un embarazo no planeado y/o una infección de transmisión sexual. Si revisamos los factores asociados con haber tenido relaciones sexuales en la adolescencia según los modelos de regresión logística presentados en la Tabla 8, vemos que en el caso de las mujeres hay mayor posibilidad en los estratos bajos y muy bajos de iniciarse sexualmente en esas edades si se compara con el estrato alto. Por el contrario, no se observan diferencias estadísticamente significativas entre el estrato medio y el alto. Paralelamente, la condición de habla indígena no resultó ser significativa para el sexo femenino. En 
Jóvenes en México: ¿existen diferencias entre hombres y mujeres en su inicio sexual y uso del condón? / C. MENKES et al.

cuanto a los estereotipos de género, los datos muestran que las jóvenes que expresaron estereotipos más tradicionales, tienen 32.3 por ciento mayor posibilidad de haber tenido relaciones sexuales en la adolescencia, si comparamos con aquellas con estereotipos menos tradicionales.

Tabla 8: Factores asociados a haberse iniciado sexualmente en la adolescencia de las mujeres de 20 a 29 años

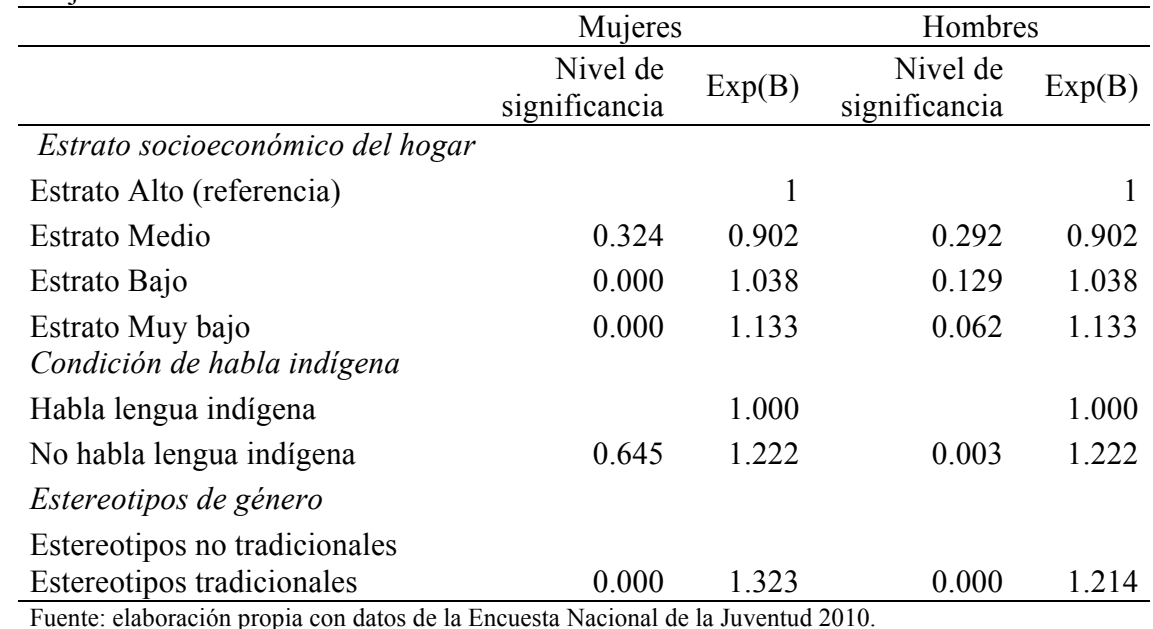

En el caso de los varones, el estrato socioeconómico del hogar no presenta una relación estadísticamente significativa con el haberse o no iniciado sexualmente en la adolescencia. Sin embargo, sí se observa una mayor posibilidad de los que no hablan lengua indígena de haber tenido relaciones sexuales en la adolescencia comparando con los que sí hablan alguna lengua indígena. Finalmente, como sucede con las mujeres, los hombres que presentaron estereotipos de género más tradicionales, también tienen mayor posibilidad de haberse iniciado sexualmente en la adolescencia.

Así, los resultados de los modelos confirman que los factores asociados a la edad de inicio sexual son diferentes en hombres y mujeres pero también que en ambos sexos los estereotipos más tradicionales se asocian con una mayor probabilidad de haberse iniciado sexualmente durante la adolescencia.

\section{USO DE MÉTODOS ANTICONCEPTIVOS EN LA PRIMERA RELACIÓN SEXUAL}

Las políticas de salud y de población han buscado combatir tanto el embarazo no planeado como las infecciones de transmisión sexual. Es por 
ello que se han intensificado en los últimos años, las campañas del uso del preservativo, dado que es el único método que prevé ambos problemas. Como se vio anteriormente, el condón femenino es poco usado, pero se decidió incluirlo en el análisis del uso del preservativo ya que junto con el masculino, son los únicos métodos que protegen tanto de las infecciones de transmisión sexual como de los embarazos no planeados.

Como se puede observar en la Tabla 9, un poco más de la mitad de los jóvenes mexicanos utiliza un método anticonceptivo en la primera relación sexual (55.6 por ciento) lo que implica que todavía cerca de 45 por ciento de los jóvenes no se protegen de ninguna manera durante su inicio sexual. El riesgo es aún mayor en el sexo femenino pues 55.5 por ciento de las jóvenes no utilizó ningún método en su primera relación sexual. El método que utilizan es fundamentalmente el condón masculino, pues 8 de cada 10 jóvenes que se protegieron lo hicieron de esta manera; el segundo lugar lo ocupa la píldora anticonceptiva. El condón femenino y la píldora anticonceptiva son utilizados de manera muy poco frecuente (Tabla 10).

Tabla 9: Distribución porcentual del uso del condón en la primera relación sexual por sexo

\begin{tabular}{lrrr}
\hline $\begin{array}{l}\text { Utilización de algún método de protección } \\
\text { o anticonceptivo en la 1ra relación sexual }\end{array}$ & $\begin{array}{c}\text { Sexo } \\
\text { Hombre }\end{array}$ & Mujer & Total \\
\hline Sí & 62.3 & 48.5 & 55.6 \\
No & 37.7 & 51.5 & 44.4 \\
Total & 100.0 & 100.0 & 100.0 \\
\hline
\end{tabular}

Fuente: elaboración propia con datos de la Encuesta Nacional de la Juventud 2010.

Tabla 10: Distribución porcentual del tipo de método usado en la primera relación sexual

\begin{tabular}{lrr}
\hline Tipo de método & Hombres & Mujeres \\
\hline Ritmo (calendario, temperatura, Billings) & 2.8 & 2.9 \\
Retiro (coito interrumpido) & 2.5 & 2.6 \\
Condón o preservativo & 78.5 & 77.4 \\
Condón femenino & 4.6 & 3.8 \\
Píldoras & 6.3 & 7.6 \\
Anticoncepción de emergencia & 3.4 & 3.4 \\
(píldora del día siguiente) & & \\
Óvulos, jaleas, espumas o diafragma & 0.2 & 0.3 \\
Dispositivo intrauterino DIU o aparato & 0.3 & 0.5 \\
Inyecciones & 1.3 & 1.4 \\
Otro & 0.1 & 0.1 \\
Total & 100.0 & 100.00 \\
\hline Fuente: elaboración propia con datos de la Encuesta Nacional de la Juventud 2010.
\end{tabular}


Jóvenes en México: ¿existen diferencias entre hombres y mujeres en su inicio sexual y uso del condón? / C. MENKES et al.

Cuando se les preguntó a los jóvenes que no usaron ningún método anticonceptivo en su primera relación sexual por qué no lo hicieron, la razón más frecuente que reportaron fue que no esperaban tener relaciones sexuales (46.9 por ciento); la segunda razón fue porque la pareja no quiso (11.2 por ciento) y, la tercera, porque no conocían los métodos anticonceptivos (10.4 por ciento) (Tabla 11).

Tabla 11: Distribución porcentual de las razones por las cuales no usaron un método anticonceptivo en la primera relación sexual

\begin{tabular}{lrrr}
\hline $\begin{array}{l}\text { Razones por las cuales no se utilizó algún } \\
\text { método anticonceptivo en la primera }\end{array}$ & \multicolumn{3}{l}{ Sexo del entrevistado } \\
relación sexual & Hombre & Mujer & Total \\
\hline Mi pareja no quiso & 6.4 & 14.9 & 11.2 \\
No esperaba tener relaciones sexuales & 56.0 & 39.9 & 46.9 \\
Yo no quería & 8.7 & 9.9 & 9.4 \\
No conocía los métodos & 11.0 & 9.9 & 10.4 \\
Me daba vergüenza conseguir los métodos & 5.7 & 4.5 & 5.0 \\
Quería un embarazo & 3.8 & 13.3 & 9.2 \\
No se siente igual & 5.4 & 3.0 & 4.1 \\
Otro & 3.0 & 4.5 & 3.8 \\
Total & 100.0 & 100.0 & 100.0 \\
\hline
\end{tabular}

Fuente: elaboración propia con datos de la Encuesta Nacional de la Juventud 2010.

Vale la pena recalcar que también se observan diferencias en este sentido de acuerdo al sexo de los jóvenes. Existe un mayor porcentaje de hombres que de mujeres que no se protegió porque no esperaba tener relaciones sexuales ( 56.9 vs. 39.9 por ciento) mientras que un mayor porcentaje de mujeres comparado con los hombres, no usó protección porque quería embarazarse (13.3 vs. 5.7 por ciento). Igualmente, más mujeres que hombres declaran que su pareja no quiso usarlo (19.9 vs. 6.4 por ciento). No cabe duda de que sobre todo en los varones, se debe de reforzar la idea de que la planeación anticipada para protegerse sexualmente, o el no estar siempre disponible cuando se presenta la oportunidad de tener relaciones sexuales, no significa ni falta de pasión ni falta de virilidad.

Tomando en cuenta una de las recomendaciones de la Conferencia de Población del Cairo en 1994, los jóvenes deben estar conscientes de que la libertad de acción y de decisión conlleva también a la responsabilidad, en este caso particular, de cuidar de sí mismos y de su pareja. Aunado a ello es claro que la sexualidad es compleja y puede asociarse muchas veces con deseos impulsivos, también los tabúes asociados con este tema, obstacu- 
lizan la posibilidad de hablar abiertamente de él, por tanto, se dificulta el planear por adelantado las estrategias de protección sexual.

El hecho que un número mayor de hombres que de mujeres no se protegieron sexualmente porque no esperaban tener relaciones sexuales, hace suponer que quizás los mandatos de género refuerzan estas actitudes en los varones, ya que deben de jugar el rol de ser impulsivos y valientes en los temas relacionados con la sexualidad, dispuestos a tener relaciones sexuales siempre que sea posible y por lo tanto, incrementan las prácticas de riesgo.

$\mathrm{Al}$ analizar la relación sexual según distintas características sociodemográficas, se constata según la Tabla 12, que a menor edad de inicio sexual, menor utilización del condón, salvo en el caso de los jóvenes que se iniciaron después de los 19 años. En efecto, resalta que de los varones que se iniciaron sexualmente entre los 12 y 14 años, únicamente 47.8 por ciento usó preservativo en la primera relación sexual y sólo 32.3 por ciento de las mujeres que se iniciaron en este rango de edad. De hecho, 64.9 por ciento de las mujeres iniciadas sexualmente entre 12 y 14 años, no usó ningún método anticonceptivo en su primera relación sexual.

Por otro lado, hay que señalar que se observa un elevado porcentaje de jóvenes que no usaron protección y se iniciaron entre 20 y 29 años, seguramente porque están menos expuestos a un embarazo no planeado. Con respecto al uso del condón según la generación de pertenencia, se observa en general una tendencia a una mayor utilización, tanto en hombres como en mujeres, en las generaciones más jóvenes.

Si se analiza por condición de habla indígena, son sorprendentes las diferencias en el uso del condón en la primera relación sexual, sobre todo en el caso del sexo femenino: 44.7 por ciento de las mujeres no indígenas se protegió en la primera relación sexual y únicamente 25.9 por ciento de las hablantes de lengua indígena.

Finalmente, con respecto al índice de estereotipos de género también se observan diferencias muy importantes en el uso del condón: los jóvenes con opiniones más tradicionales se protegieron en menor medida que los jóvenes con opiniones menos tradicionales. Las diferencias más importantes se dan en las mujeres, ya que las que mostraron tener opiniones más tradicionales con respecto a los roles de género son las que menos se protegieron: únicamente 31.1 por ciento de las que expresaron opiniones más tradicionales contra 55.5 por ciento de las mujeres que expresaron conceptos menos tradicionales, usaron el condón en la primera relación sexual. 
Jóvenes en México: ¿existen diferencias entre hombres y mujeres en su inicio sexual y uso del condón? / C. MENKES et al.

Tabla 12: Porcentaje del uso del condón en la primera relación sexual de jóvenes mexicanos

\begin{tabular}{lrrrrrrr}
\hline & \multicolumn{4}{c}{ Hombres } & \multicolumn{4}{c}{ Mujeres } \\
\cline { 2 - 7 } $\begin{array}{l}\text { Edad de la primera } \\
\text { relación sexual }\end{array}$ & $\begin{array}{r}\text { Uso del } \\
\text { condón }\end{array}$ & $\begin{array}{r}\text { Uso de otro } \\
\text { método }\end{array}$ & $\begin{array}{r}\text { No usó } \\
\text { ningún método }\end{array}$ & $\begin{array}{rrrr}\text { Usó del } \\
\text { condón }\end{array}$ & $\begin{array}{r}\text { Uso de otro } \\
\text { método }\end{array}$ & $\begin{array}{r}\text { No usó } \\
\text { ningún método }\end{array}$ \\
\hline 12-14 años & 47.8 & 4.9 & 47.3 & 32.3 & 2.8 & 64.9 \\
15 a 17 años & 57.9 & 5.9 & 36.2 & 42.8 & 4.5 & 52.7 \\
18 a 19 años & 64.2 & 5.3 & 30.5 & 55.7 & 5.0 & 39.3 \\
20 a 29 años & 49.4 & 5.6 & 45.0 & 46.4 & 5.3 & 48.2
\end{tabular}

Porcentaje del uso del condón en la primera relación sexual según grupo de edad actual de jóvenes mexicanos

Estratos socioeconómicos

$\begin{array}{lllllll}\text { Muy Bajo } & 43.8 & 3.8 & 52.4 & 24.6 & 4.5 & 70.9 \\ \text { Bajo } & 50.7 & 5.2 & 44.1 & 34.7 & 4.3 & 61.0 \\ \text { Medio } & 57.2 & 4.4 & 38.4 & 46.8 & 5.6 & 47.6 \\ \text { Alto } & 63.9 & 5.0 & 31.1 & 57.8 & 5.4 & 36.8\end{array}$

Porcentaje de uso del condón según la condición de indigenismo en la primera relación sexual de jóvenes mexicanos

Hablante de lengua indigena

$\begin{array}{lllllll}\text { Sí } & 42.5 & 5.8 & 51.7 & 25.9 & 2.8 & 71.4 \\ \text { No } & 57.3 & 5.6 & 37.1 & 44.7 & 4.6 & 50.7\end{array}$

Porcentaje del uso del condón de los jóvenes de 12 a 29 años en la primera relación sexual según índice de género y sexo

Índice de género

Tradicional

$51.1 \quad 5.1$

$43.9 \quad 31.1$

4.1

64.8

No tradicional

$60.4 \quad 6.0$

$33.7 \quad 55.5$

5.2

Porcentaje del uso del condón en la primera relación sexual según grupo de edad actual de jóvenes mexicanos

Generación

\begin{tabular}{lllllll}
$12-14$ años & 65.7 & 2.9 & 31.4 & 47.8 & 3.2 & 49.0 \\
15 a 19 años & 62.5 & 6.5 & 31.0 & 53.7 & 4.1 & 42.2 \\
20 a 24 años & 57.6 & 5.6 & 36.8 & 46.3 & 4.2 & 49.5 \\
25 a 29 años & 51.6 & 5.0 & 43.4 & 36.9 & 5.2 & 57.9 \\
\hline Fuente: elaboración propia con datos de la Encuesta Nacional de la Juventud 2010
\end{tabular}




\section{FACTORES SOCIODEMOGRÁFICOS ASOCIADOS AL USO DEL CONDÓN} EN EL INICIO SEXUAL

Con el fin de conocer la probabilidad de asociación de factores sociodemográficos con el uso del preservativo, se estimaron dos modelos de regresión logística (Tabla 13). Los resultados muestran que estos factores sociodemográficos se relacionan de manera importante con el uso del preservativo en la primera relación sexual. Tanto en hombres como en mujeres, el hecho de que el estrato socioeconómico tenga una razón de momios mayor a uno y que va aumentando conforme crece el estrato socioeconómico, muestra que a medida que mejoran las condiciones socioeconómicas de los miembros del hogar, se incrementa considerablemente la probabilidad de que los jóvenes usen condón en la primera relación sexual (Tabla 13).

En los varones la probabilidad que en el estrato bajo y medio usen condón es 20.7 y 52.8 por ciento mayor que en el estrato Muy bajo y en las mujeres es mayor en 34.2 y 85.6 por ciento, respectivamente. La probabilidad aumenta todavía más en el estrato Alto, ya que la probabilidad que los varones del estrato Alto usen condón es 85.9 por ciento mayor que la del estrato Muy bajo, y en las mujeres se multiplica más de dos veces (2.3) la posibilidad de uso en el estrato Alto respecto al Muy bajo. Al parecer el estrato socioeconómico al que pertenecen los jóvenes se relaciona de manera muy importante con la protección sexual, de todas formas el contexto socioeconómico parece tener todavía mayor impacto en el caso del sexo femenino.

En cuanto a la condición de habla indígena, ésta no mostró una relación significativa con el uso del preservativo en el modelo, es muy probable que esto se deba al reducido número de jóvenes de hablante indígena captados en la encuesta. Respecto al índice de género, los varones que expresaron opiniones no tradicionales en torno a los roles de género tienen 27.5 por ciento mayor posibilidad de protegerse con un preservativo en su primera relación sexual que los que expresaron opiniones tradicionales y las mujeres con opiniones menos tradicionales tienen 56.7 por ciento mayor posibilidad de protección sexual respecto a las jóvenes con opiniones tradicionales. En cuanto a la generación de pertenencia, el que la razón de momios sea menor a uno, muestra que los varones y las mujeres de generaciones más viejas tienen menor probabilidad de usar condón en su inicio sexual que los más jóvenes. 
Jóvenes en México: ¿existen diferencias entre hombres y mujeres en su inicio sexual y uso del condón? / C. MENKES et al.

Tabla 13: Factores asociados al uso de condón en los jóvenes en su iniciación sexual por sexo (Modelo de regresión logística)

\begin{tabular}{|c|c|c|c|c|}
\hline \multirow{2}{*}{$\begin{array}{l}\text { Sexo } \\
\text { Características sociodemográficas }\end{array}$} & \multicolumn{2}{|c|}{ Hombres } & \multicolumn{2}{|c|}{ Mujeres } \\
\hline & Sig. & $\begin{array}{r}\text { Razón de } \\
\text { momios }\end{array}$ & Sig. & $\begin{array}{r}\text { Razón de } \\
\text { momios }\end{array}$ \\
\hline \multicolumn{5}{|l|}{ Estrato socioeconómico } \\
\hline Muy bajo & & 1 & & 1 \\
\hline Bajo & 0.033 & 1.207 & 0 & 1.392 \\
\hline Medio & 0 & 1.528 & 0 & 2.135 \\
\hline Alto & 0 & 1.859 & 0 & 3.032 \\
\hline \multicolumn{5}{|l|}{ Hablante de Lengua indígena } \\
\hline Indígena & & 1 & & 1 \\
\hline No Indígena & 0.084 & 0.794 & 0.265 & 0.839 \\
\hline \multicolumn{5}{|l|}{ Tamaño de localidad } \\
\hline Rural & 1 & 1 & & 1 \\
\hline Urbana & 0 & 1.233 & 0 & 1.626 \\
\hline \multicolumn{5}{|l|}{ Índice de género } \\
\hline Tradicional & & 1 & & 1 \\
\hline No tradicional & 0 & 1.305 & 0 & 1.657 \\
\hline \multicolumn{5}{|l|}{ Generación de pertenencia } \\
\hline Generación 1981-1985 & & 1 & & 1 \\
\hline Generación 1996-1998 & 0.289 & 0.694 & 0.012 & 0.393 \\
\hline Generación 1991-1995 & 0.029 & 0.47 & 0 & 0.253 \\
\hline Generación 1990-1986 & 0.004 & 0.368 & 0 & 0.163 \\
\hline \multicolumn{5}{|l|}{ Edad primera relación sexual } \\
\hline 15 a 19 años & 0 & 1.522 & 0 & 1.851 \\
\hline 20 a 29 años & 0.047 & 1.286 & 0 & 2.234 \\
\hline $\begin{array}{l}\text { Edad de la pareja sexual } \\
\text { Edad de } 12 \text { a } 14 \text { años }\end{array}$ & & 1 & & 1 \\
\hline 15 a 19 años & 0 & 1.451 & 0.717 & 1.09 \\
\hline 20 a 29 años & 0.001 & 1.47 & 0.994 & 1.002 \\
\hline Constante & 0.332 & 0.714 & 0.072 & 0.478 \\
\hline
\end{tabular}

Fuente: elaboración propia con datos de la Encuesta Nacional de la Juventud 2010.

En cuanto a la edad de inicio sexual, el modelo muestra claramente que conforme aumenta la edad de inicio sexual, crece la probabilidad de 
protegerse con el preservativo tanto en hombres como en mujeres. Paralelamente, el modelo muestra que crece considerablemente el uso del condón cuando los varones se inician con parejas sexuales de mayor edad, mientras que en el caso de las mujeres la edad de la pareja sexual no fue significativa. Finalmente se observa que en los varones aumenta la probabilidad del uso de un preservativo si se inician con la novia $(\mathrm{RM}=1.219)$ y disminuye si el debut sexual es con la esposa $(\mathrm{RM}=0.364)$, comparando con el inicio sexual con una amiga. Llama la atención que en el caso del sexo masculino sea menor con la amiga que con la novia, pues uno esperaría que exista mayor confianza con esta última, pero es probable que esto se deba a la menor posibilidad de planear el primer encuentro sexual con una amiga. En el caso de las mujeres no se encuentran diferencias estadísticamente significativas en el uso si se inician con el amigo o con el novio, pero sí disminuye de manera muy importante el uso si se inician con el esposo $(\mathrm{RM}=0.343)$.

Se puede concluir según los modelos, que el uso del preservativo para protegerse tanto de un embarazo no deseado como de las infecciones de transmisión sexual en los jóvenes mexicanos, disminuye en los estratos socioeconómicos más desfavorecidos, en las zonas rurales, en las generaciones más viejas, en los que se inician a edades muy tempranas, en los que se inician sexualmente con el esposo/a, y en los que presentan estereotipos de género tradicionales. La mayor edad de la pareja sexual sólo aumenta la protección sexual en el caso de los varones.

\section{Conclusiones}

El trabajo refuerza los hallazgos de la literatura relativos a las prácticas sexuales, la sexualidad y las dinámicas de género en éstos ámbitos (Sosa 2005 y 2010; Geldstein y Pantelides, 1997; Fernández, 2013; Spreacher y Regan, 2001; Warr, 2001), evidenciando el carácter altamente generizado de las prácticas sexuales entre los y las adolescentes mexicanos (Quintana y Vásquez, 1999; Aguirre y Güell, 2002; Sprecher y Regan, 2001; Menkes y Suárez, 2003; De Jesús y Menkes, 2016). Los resultados refrendan que los varones se inician sexualmente a edades más tempranas que las mujeres. El inicio sexual fue diferente según el sexo, no sólo por lo anterior, sino también porque las mujeres declararon haberse iniciado principalmente con el novio y por amor, mientras que una buena parte de los varones se inició con amigas y no pocas veces, por curiosidad. El compañero sexual de las mujeres siempre fue mayor que el de los hombres en cualquier contexto. 
Jóvenes en México: ¿existen diferencias entre hombres y mujeres en su inicio sexual y uso del condón? / C. MENKES et al.

Probablemente la mayoría de las mujeres solteras declararon haberse iniciado con el novio sugiriendo el importante peso de las imágenes y discursos en torno al amor romántico como marco idóneo de las relaciones sexuales para las mujeres, lo que si acaso cuestiona el esquema tradicional del matrimonio, continúa reproduciendo las desigualdades de género en el ámbito de la sexualidad y las relaciones sexuales. En el polo opuesto, los varones reafirman su virilidad y dominancia al declarar, sea cierto o no, que a edad temprana tuvieron relaciones sexuales con novias y/o amigas. Esto se relaciona, como lo han sugerido algunos estudios previos (Warr, 2001; Sosa y Menkes, 2003; De Jesús y Menkes 2014), con la protección sexual, ya que las jovencitas suelen asociar el amor romántico con la confianza y a su vez, la confianza con el amor, y por lo tanto con la imposibilidad de percibir , o expresar la idea, de que la pareja sexual pueda significar un riesgo. De manera que la posibilidad de hablar de la protección sexual puede obstaculizarse. Sobra decir que los mandatos de género también presionan a los jóvenes varones para tener relaciones sexuales siempre que sea posible, aunque no estén preparados para protegerse sexualmente.

Convendría que las políticas de población y de salud tomaran en cuenta que persisten diferencias muy marcadas según el sexo tanto en la edad de inicio sexual, como en las características de la pareja y en los significados que le dan los jóvenes a su inicio sexual. Además de lo dicho anteriormente, vale añadir que el principal método de protección sexual utilizado fue el preservativo masculino y que los varones se protegieron con mayor frecuencia que las mujeres en su primera relación sexual. La principal razón por la cual los jóvenes no usaron un método de protección en su inicio fue por no haber planeado un encuentro sexual, lo que refuerza la necesidad de eliminar tabús en torno a la sexualidad de los jóvenes con el fin de que por iniciativa propia adquieran un preservativo. Es vital concientizar a los adolescentes de la importancia de estar preparados para una mejor protección sexual.

El modelo estadístico muestra que la protección sexual a través del preservativo en los jóvenes mexicanos es menor en las mujeres, en los estratos socioeconómicos más desfavorecidos, en las zonas rurales, en la población indígena, en las generaciones más viejas, en los que se inician a edades muy tempranas y en quienes presentan estereotipos de género tradicionales. Uno de los hallazgos más importantes en este estudio es haber mostrado que los jóvenes con estereotipos de género menos tradicionales no incrementan la probabilidad de iniciarse sexualmente a edad más temprana, 
pero tienen en cambio mayor probabilidad de protegerse de un embarazo no planeado, así como de evitar contraer una infección sexual.

Los resultados permiten afirmar como ya Stewart (1999) ha señalado, que las prácticas de sexo protegido (que involucran el uso de condón) implican un cambio paradigmático en las identidades de género y por tanto el desmantelamiento de las relaciones de género prevalecientes en la sociedad mexicana. Desde esta perspectiva, se sugiere como un eje central promover la auto apropiación de los cuerpos de las mujeres promoviendo a su vez nociones incorporadas (embodied) del erotismo sexual (erotic sex) (Warr, 2001) y del placer como componentes centrales de la salud sexual (Pick, Givaudan y Kline, 2005; Fine, 1999).

Esto significa reconocer que el sexo protegido involucra complejos procesos de negociación sexual, que requieren un grado de comunicación abierta sobre el propio deseo sexual, lo cual no es fácilmente accesible en sociedades como la mexicana donde las relaciones sexuales heterosexuales están marcadas por las desigualdades de género y poder. Igualmente, es importante tener en cuenta que las prácticas de sexo protegido y la prevención de embarazos se desprenden no sólo de elecciones individuales e interacciones interpersonales, sino también de sistemas más amplios de significados morales y coacciones sociales (Gammeltof, 2002). Lo antes mencionado implica cuestionar el tradicional abordaje en las campañas de salud que tienden a simplificar la opción del uso de condón como una práctica y una elección aproblemática e individual y no como una práctica socialmente condicionada que adquiere sentido en relación al contexto social en el que tiene lugar (Wheathersley, 2015; O'brien et al., 2013; Rodríguez, 2008 y 2014).

Sin duda en las políticas de educación sexual de los jóvenes deben intensificarse los cuestionamientos de los roles tradicionales masculinos y femeninos, además de alentar la reflexión y la construcción colectiva de la equidad de género. Estos hallazgos cuestionan las creencias más conservadoras que argumentan que la equidad de género lleva a los adolescentes a una mayor permisividad sexual, pues cabe subrayarlo una vez más, no se encontró un aumento en la probabilidad de inicio sexual, pero sí se mostró que se incrementa la probabilidad de usar un preservativo, es decir, que los jóvenes practiquen una sexualidad más protegida.

Si bien se ha dado un avance en el conocimiento y uso del condón en nuestro país y se ha dado una mayor apertura en el comportamiento sexual de los adolescentes, aún persisten fuertes obstáculos que limitan a los jó- 
Jóvenes en México: ¿existen diferencias entre hombres y mujeres en su inicio sexual y uso del condón? / C. MENKES et al.

venes para gozar de una sexualidad protegida, sobre todo en los contextos socioeconómicos más desfavorecidos de nuestro país.

\section{LIMITACIONES METODOLÓGICAS DEL ESTUDIO}

Una limitación de los estudios cuantitativos sobre la sexualidad se refiere a que lo que declaran los jóvenes acerca de su sexualidad no siempre es verdadero; se especula que las mujeres solteras suelen ocultar sus prácticas sexuales por presiones sociales y los varones a exagerar el número de encuentros sexuales. Sin embargo, la información de las encuestas nacionales ha sido bastante consistente, por lo que se sostiene la utilidad del análisis de esta información.

Otra limitación se refiere a que los estudios sobre sexualidad consideran que ésta no sólo se expresa cuando ocurre el coito, sino desde que se manifiesta desde la infancia de múltiples maneras, lo que es difícil de aprehender en las encuestas cuantitativas. Además, en la mayoría de las encuestas nacionales, incluidas la Encuesta Nacional de la Juventud, no se especifica a qué tipo de relación sexual se está aludiendo y por tanto, no se sabe con precisión los riesgos de embarazo y de transmisión de infecciones sexuales.

Se asume que varias de las características sociodemográficas presentadas en este estudio como son el estrato socioeconómico, la condición de habla indígena, el tamaño de localidad, o las mismas opiniones respecto a los roles de género, son las mismas que cuando los jóvenes tuvieron su primera relación sexual. Esto debido a que no existe una historia de estas características, únicamente se cuenta con la información en el momento en que se hizo la encuesta.

Otra limitación se refiere a que no se cuenta con una historia de las relaciones sexuales de los jóvenes. Se decidió centrarse en la primera relación sexual, ya que varios estudios han mostrado que una vez que los jóvenes se protegen en el primer encuentro sexual, la probabilidad de que se protejan posteriormente aumenta de manera considerable, pues significa que ya rompieron con muchos de los obstáculos y tabúes que conlleva la protección sexual.

En el mismo sentido, podemos afirmar que no contamos con una historia de uniones en la encuesta por lo que no podemos saber si el joven se encontraba unido o casado en su primera relación sexual, aunque afortunadamente sí se puede saber cuál era el tipo de relación que tuvo con su primera pareja sexual (amigo, novio, esposo, etcétera) 
Cabe aclarar que la ENJ 2010 no fue realizada con fines de mediciones directamente demográficas, pero sí constituye una de las pocas encuestas nacionales que proporciona temáticas relacionadas con la sexualidad de hombres y mujeres y además nos permite contar con las opiniones de los jóvenes respecto a distintas temáticas, para este caso en particular respecto a los roles de género, por lo que fue importante explotar dicha información y relacionarla con las prácticas y la protección sexual. Es importante señalar que como esta encuesta fue coordinada por el Centro Regional de Investigaciones Multidisciplinarias, nos fue posible participar en la elaboración de las preguntas sobre sexualidad y género, lo que nos permitió profundizar en esta temática. Sería importante contar con una encuesta más reciente que incluya a los jóvenes varones y a las mujeres y poder así, continuar con estas perspectivas de análisis.

\section{REFERENCIAS BibLIOGRÁFICAS}

Aguirre, Rodrigo y Güell, Pedro, 2002, Hacerse hombres. La construcción de la masculinidad en los adolescentes y sus riesgos. OPS, OMS, UNFPA, Asdi.

Amuchástegui, Ana y Aggleton, Peter, 2007, "I Had a guilty conscience because i wasn't going to marry her: ethical dilemmas for Mexican men in their sexual relationships with women", en Sexualities, vol. 10, núm. 1, pp. 61-81.

Amuchástegui, Ana, 2007, "Ética deseo y masculinidad: la difícil relación entre lo sexual y lo reproductivo", en Amuchástegui, A. e Szasz, I. (coord.). Sucede que me canso de ser hombre. Relatos y reflexiones sobre hombres y masculinidades en México. Colmex: México.

Cáceres, Carlos, 1999, La (re)configuración del universo sexual: cultura(s) sexual(es) y salud sexual entre los jóvenes de Lima a vuelta de milenio, Lima, Universidad Peruana Cayetano Heredia y REDESS Jóvenes, 147 p.

Echarri Cánovas, Carlos Javier, 2008, "Desigualdad socioeconómica y salud reproductiva: una propuesta de estratificación social aplicable a las encuestas", en Lerner, Susana y Szasz, Ivonne (eds.), Salud reproductiva y condiciones de vida en México, Tomo I, México, El Colegio de México, pp. 59-113.

Echarri Canóvas, Carlos Javier, 2016, “Anexo metodológico”, en Chávez Ana María, Corona Vázquez Rodolfo, Echarri Canóvas Carlos Javier (eds.), Los jóvenes mexicanos en la encrucijada de 2010, Cuernavaca, CRIM-UNAM, pp. 445-450.

Fernández, Ana, 2013, Las lógicas sexuales, amor política, y violencias, Nueva visión: Buenos Aires. 
Jóvenes en México: ¿existen diferencias entre hombres y mujeres en su inicio sexual y uso del condón? / C. MENKES et al.

Fine, Michelle, 1999, "Sexualidad, educación y mujeres adolescentes. El discurso ausente del deseo", en Belausteguigoitia, M., Géneros Prófugos. Feminismo y Educación. PUEG/UNAM, México, pp. 291-321.

Fraser, A. M., Brockert J.E. y Ward RH., 1995, “Association of young maternal age with adverse reproductive outcomes", en New England Journal of Medicine, 332(17), 1113-1117.

Gammeltof, Tine, 2002, "Seeking trust and transcendence: sexual risk-taking among Vietnamese youth"., en Social Science and Medicine, núm. 55, pp. 484496.

Gayet, Cecilia y Solís, Patricio, 2007, "Sexualidad saludable de los adolescentes: la necesidad de políticas basadas en evidencias", en Salud Pública de México, vol. 49, Edición especial 1, pp. E47-E51.

Gayet, Cecilia; Juárez, Fátima; Pedrosa, Laura A. y Magis, Carlos, 2003, "Uso del condón entre adolescentes mexicanos para la prevención de las infecciones de transmisión sexual”, en Salud Pública de México, vol. 45(sup. 5):632-640.

Geldstein, Rosa y Pantélides, Edith, 1997, "Double subordination, double risk: class, gender and sexuality in adolescent women in Argentina", en Reproductive Health Matters, núm. 9, pp. 34-52.

Giordano, Francesca; Thumme, Beverly y Sierra, Georgina, 2009, "The hopes and dreams of Honduran women regarding their daughters' sexuality", en Qualitative Health Research, vol. 19, núm. 7, pp. 996-1009.

Instituto Mexicano de la Juventud, 2010, Encuesta Nacional de la Juventud, 2010, México, Instituto Mexicano de la Juventud.

Instituto Nacional de Salud Pública, 2000, Encuesta Nacional de Salud, 2000, México, Instituto Nacional de Salud Pública.

Instituto Nacional de Salud Pública, 2012, Encuesta Nacional de Nutrición y Salud, 2012, México, Instituto Nacional de Salud Pública.

Jesús-Reyes de, David y Menkes-Bancet, Catherine, 2016, Salud reproductiva de los estudiantes de escuelas públicas de educación media y media superior de Nuevo León. México: Universidad Nacional Autónoma de México.

Jesús-Reyes de, David y González Almontes, Esmeralda, 2014, "Elementos teóricos para el análisis del embarazo adolescente", en Sexualidad, Salud y Sociedad-Revista Latinoamericana, núm. 17, 98-123.

Jesús-Reyes de, David y Menkes-Bancet, Catherine, 2014, "Prácticas y significados del uso del condón en varones adolescentes de dos contextos de México", en Papeles de Población, vol. 20, núm. 79, enero-marzo, pp. 73-97.

Jones, Daniel, 2010, Sexualidades adolescentes. Amor, placer y control en la Argentina contemporánea. CLACSO-CICCUS.

Juárez, Fátima y Gayet, Cecilia, 2005, "Salud sexual y reproductiva de los adolescentes en México: un nuevo marco de análisis para la evaluación y diseño de políticas", en Papeles de población, vol. 11 núm. 45 , pp. 177-219. 
Juárez, Fátima; Palma, José Luis; Singh, Susheela y Bankole, Akinrinola, 2010, Las necesidades de salud sexual y reproductiva de las adolescentes en México: Retos y Oportunidades, Nueva York, Guttmacher Institute, 35 p.

Keijzer de, Benno, 1999, "Los derechos sexuales y reproductivos desde la dimensión de la masculinidad”, en México diverso y desigual. Enfoques sociodemográficos. V Reunión de Investigación Sociodemográfica. vol. 4. Colmex, pp.307-318.

Lamas, Marta, 2003, "Maternidad y violencia simbólica" en Uribe, R. y Billings, D. Violencia sobre la salud de las mujeres. México FEMEGO,-IPAS, pp. 114-121.

Mendoza, Doroteo; Sánchez Castillo, Miguel; Hernández López, María Felipa y Mendoza García, Ma Eulalia, 2009, "35 años de planificación familiar en México" en CONAPO, La situación demográfica en México 2009, México, CONAPO, pp. 39-52.

Menkes, Catherine y Serrano, Olga, 2010, "Embarazo adolescente en México: niveles y condicionantes sociodemográficos" en Chávez, Ana María y Menkes, Catherine (eds.), Procesos y Tendencias Poblacionales en el México contemporáneo. Una mirada desde la ENADID 2006, Cuernavaca, CRIM/UNAM y Secretaría de Salud, pp. 182-199.

Menkes, Catherine y Suárez, Leticia, 2003, "Sexualidad y embarazo adolescente en México", en Papeles de Población, vol. 9, núm. 35, pp.1-31.

Menkes, Catherine, 2016, "Opciones de vida de la juventud y reproducción temprana en México", en Ponencia presentada en la plenaria La reproducción temprana y las opciones de vida de la juventud en la XIII Reunión Nacional de Investigación Demográfica en México. Dinámicas de población y desigualdad, Sociedad Mexicana de Demografía, Cd. de México.

O’Brien, Colleen; Chumbler, Neale; Bute, Jennifer y Huff, Amber, 2013, “ Building a "Better Life": The Transformative Effects of Adolescent Pregnancy and Parenting", en SAGE Open, pp. 1-9.

Oliva, Ana; Serra, Leo y Vallejo, Rodolfo, 1997, "Patrones de comportamiento sexual y contraceptivo durante la adolescencia”, en Infancia Aprendida, núm. 77, pp. 19-34.

Palma, Yolanda y González, Eduardo, 2010, “Iniciación sexual. Análisis de la ENADID 2006", en Ana María Chávez y Menkes Catherine (eds.), Procesos y tendencias poblacionales en el México contemporáneo. Una mirada desde la Enadid 2006, Cuernavaca, CRIM/UNAM y Secretaría de Salud, pp. 50-74.

Pick, S.; Givaudan, M. y Kline, K. F., 2005, “Sexual pleasure as a key component of integral sexual health" in Feminism and Psychology, vol. 15, pp. 44-49.

Quintana, Alicia y Vásquez, Ernesto, 1999, Construcción Social de la Sexualidad Adolescente: Género y Salud Sexual. Instituto de Educación y Salud, Lima, Perú.

Rodríguez, Gabriela, 2002, "Magia, religiosidad y pragmatismo en la sexualidad de jóvenes rurales”, en Figueroa, Juan G. (coord.). Ética, religión y reproducción. Católicas por el derecho a decidir, México, pp.119-131. 
Jóvenes en México: ¿existen diferencias entre hombres y mujeres en su inicio sexual y uso del condón? / C. MENKES et al.

Rodríguez, Gabriela, 2008, "Introducción”, en Stern (coord.). Adolescentes en México. Investigación, experiencia y estrategias para mejorar su salud sexual y reproductiva, Colmex.

Rodríguez, Jorge, 2008, Reproducción adolescente y desigualdades en América Latina y el Caribe: un llamado a la reflexión y a la acción. CELADE, CEPAL.

Rodríguez, Jorge, 2014, La reproducción en la adolescencia y sus desigualdades en América Latina. Introducción al análisis demográfico, con énfasis en el uso de microdatos censales de la ronda de 2010. UNFPA-CEPAL.

Romero, María, 2006, El comportamiento sexual y reproductivo de los adolescentes y jóvenes mexicanos asociados a nuevas variables educativas, 2000. Tesis de maestría del Colegio de la Frontera Norte.

Sosa-Sánchez, Itzel y Menkes, Catherine, 2003, “Algunas reflexiones acerca de los obstáculos en el uso del condón. Un estudio en Morelos", ponencia presentada en la VII Reunión de Investigación Demográfica en México, 2-5 de diciembre, México.

Sosa-Sánchez, Itzel, 2005, Los significados de la salud y la sexualidad en jóvenes. Un estudio de caso en escuelas públicas en Cuernavaca. México, INMUJERES.

Sosa-Sánchez, Itzel, 2010, Significados del cuerpo y del riesgo en el marco de la sexualidad y de la reproducción: un estudio de caso con jóvenes en Cuernavaca, Tesis de Maestría, FLACSO-CLACSO: Buenos Aires.

Spreacher, Susan y Regan, Pamela, 2001, "College Virgins: how men and women perceive their sexual status", en Kenneth Davidson et al. (ed.). Speaking of sexuality: interdisciplinary readings. Roxbury Publishing, pp. 112-123.

Stern, Claudio y Menkes, Catherine, 2012, "Embarazo adolescente y estratificación social", en Stern Claudio (Coord.) El "problema" del embarazo en la adolescencia: contribuciones a un debate, Centro de Estudios Sociológicos El Colegio de México, México pp 227-274.

Stewart, Fiona, 1999, “Once you get a reputation, your life's like.... wrecked: the implications of reputation for young women's sexual health and well being", en Social Science and Medicine, Pergamon, Women's Studies International Forum, vol. 22, núm. 3, pp. 373-383, USA.

Szasz, Ivonne, 1998, "Sexualidad y género: algunas experiencias de investigación en México", en Debate feminista, año 9, vol. 18, octubre, México pp. 77-104.

Ulanowicz, M.; Parra, K.; Wendler, G. y Tisiana Monzón, L., 2006, "Riesgo en el embarazo adolescente", en Revista de Posgrado de la VIa Cátedra de Medicina, núm. 153: 13- 17

Warr, Deborah, 2001, "The Importance of Love and Understanding Speculation on Romance in Safe Sex Health". Women's Studies International Forum, vol. 24, núm. 2, pp.241 - 252.

Weatherley, Richard, 2015, “Teenage pregnancy, professional agendas, and problem definitions", en The Journal of Sociology and Social Welfare, 14(2): 6-35. 
Welti, Carlos, 2005, "Inicio de la vida sexual y reproductiva", en Papeles de Población, vol. 11, núm. 45, pp. 143-176.

\section{RESUMEN CURRICULAR DE LOS AUTORES}

\section{Catherine Menkes Bancet}

Es Licenciada en Economía por la Universidad Autónoma Metropolitana, Unidad Iztapalapa, Maestra en Demografía por El Colegio de México y Doctora en Ciencias Políticas y Sociales con especialidad en Sociología por el Centro de Investigación y Docencia en Humanidades del Estado de Morelos. Sus temas de investigación hacen referencia a procesos migratorios, fecundidad y migración, así como a temáticas relacionadas con la salud reproductiva. Ha escrito múltiples libros y publicado en diversas revistas nacionales e internacionales, así como dirigido proyectos de investigación financiados por organismos de carácter internacional. Actualmente es investigadora del Centro Regional de Investigaciones Multidisciplinarias de la Universidad Nacional Autónoma de México (CRIM-UNAM) y pertenece al Sistema Nacional de Investigadores Nivel II.

Dirección electrónica: menkes@unam.mx

\section{David de Jesús Reyes}

Es Licenciado en Ciencia Política por la Universidad Autónoma Metropolitana Unidad Iztapalapa, Maestro en Estudios de Población por el Centro Regional de Investigaciones Multidisciplinarias de la Universidad Nacional Autónoma de México y Doctor en Filosofía con Orientación en Políticas de Bienestar Social por la Universidad Autónoma de Nuevo León. Sus líneas de investigación son la epidemiología sociocultural, sexualidad y reproducción, salud reproductiva, género, masculinidad y diversidad sexual. Ha escrito artículos en revistas nacionales e internacionales, así como libros y capítulos de libro, siendo también responsable de proyectos de investigación en sexualidad y reproducción adolescente. Actualmente se desempeña como Profesor-Investigador en el Instituto de Investigaciones Sociales, de la Universidad Autónoma de Nuevo León y pertenece al Sistema Nacional de Investigadores Nivel I.

Dirección electrónica: jesusreyes@unam.mx 
Jóvenes en México: ¿existen diferencias entre hombres y mujeres en su inicio sexual y uso del condón? / C. MENKES et al.

\section{Itzel A. Sosa Sánchez}

Becaria del Programa de Becas posdoctorales en la UNAM, Centro Regional de Investigaciones Multidisciplinarias, UNAM. Doctora en sociología por la Universidad Laval (Québec, Canadá). Maestra en teorías y metodologías de las ciencias sociales (FLACSO, sede Buenos Aires) y Maestra en estudios de población por el CRIM-UNAM (México). Sus líneas de investigación se centran en el género, la reproducción, la sexualidad y hermenéutica del cuerpo. Ha escrito múltiples libros y publicado en diversas revistas nacionales e internacionales. Actualmente es investigadora del Centro Regional de Investigaciones Multidisciplinarias de la Universidad Nacional Autónoma de México (CRIM-UNAM) y pertenece al Sistema Nacional de Investigadores Nivel I.

Dirección electrónica: Itzela@correo.crim.unam.mx 